\title{
Erratum to: The L450P mutation in KCND3 brings spinocerebellar ataxia and Brugada syndrome closer together
}

\author{
A. Duarri ${ }^{1}$ - E. Nibbeling ${ }^{1} \cdot$ M. R. Fokkens ${ }^{1} \cdot$ M. Meijer $^{2}$ - E. Boddeke ${ }^{2}$.

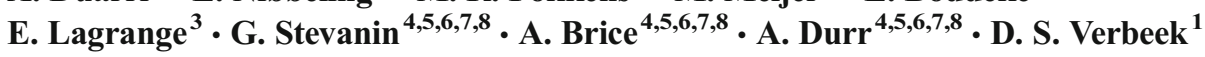

Published online: 12 April 2015

(C) Springer-Verlag Berlin Heidelberg 2015

Erratum to: Neurogenetics. 2013 Nov;14(3-4):257-8.

DOI 10.1007/s10048-013-0370-0. Epub 2013

Aug 21.

In the title L450P should have been L450F. The same error appeared in the second and tenth sentence of the fourth paragraph. These errors appeared in the version of the paper published in 2013 Nov;14(3-4):257-8. The authors regret the errors.

The online version of the original article can be found at http://dx.doi.org/ 10.1007/s10048-013-0370-0.

D. S. Verbeek

d.s.verbeek@umcg.n

1 Department of Genetics, University Medical Center Groningen, University of Groningen, Groningen, The Netherlands

2 Department of Medical Physiology, University Medical Center Groningen, University of Groningen, Groningen, The Netherlands

3 Reference Center for Rare Neuromuscular Diseases, Pôle de psychiatrie et neurologie, CHU de Grenoble, Grenoble, France

4 INSERM, U975, 75013 Paris, France

5 Université Pierre et Marie Curie-Paris 6, UMR-S975, Centre de Recherche de l'Institut du Cerveau et de la Moelle épinière, Groupe Hospitalier Pitié-Salpêtrière, 75013 Paris, France

6 CNRS, UMR 7225, 75013 Paris, France

7 Département de Génétique et Cytogénétique, AP-HP, Groupe, Hospitalier Pitié-Salpêtrière, 75013 Paris, France

8 ICM, Brain and Spine Institute, University Salpêtrière Hospital, Paris, France 